\title{
Existence of exotic torus configuration in high-spin excited states of ${ }^{40} \mathrm{Ca}$
}

\author{
T. Ichikawa, ${ }^{1}$ J. A. Maruhn, ${ }^{2}$ N. Itagaki, ${ }^{1}$ K. Matsuyanagi, ${ }^{1,3}$ P.-G. Reinhard, ${ }^{4}$ and S. Ohkubo ${ }^{5,6}$ \\ ${ }^{1}$ Yukawa Institute for Theoretical Physics, Kyoto University, Kyoto 606-8502, Japan \\ ${ }^{2}$ Institut fuer Theoretische Physik, Universitaet Frankfurt, D-60438 Frankfurt, Germany \\ ${ }^{3}$ RIKEN Nishina Center, Wako 351-0198, Japan \\ ${ }^{4}$ Institut für Theoretische Physik, Universität Erlangen, D-91058 Erlangen, Germany \\ ${ }^{5}$ Department of Applied Science and Environment, University of Kochi, Kochi 780-8515, Japan \\ ${ }^{6}$ Research Center for Nuclear Physics, Osaka University, Ibaraki, Osaka 567-0047, Japan
}

(Dated: July 31, 2018)

\begin{abstract}
We investigate the possibility of the existence of the exotic torus configuration in the high-spin excited states of ${ }^{40} \mathrm{Ca}$. We here consider the spin alignments about the symmetry axis. To this end, we use a threedimensional cranked Skyrme Hartree-Fock method and search for stable single-particle configurations. We find one stable state with the torus configuration at the total angular momentum $J=60 \hbar$ and an excitation energy of about $170 \mathrm{MeV}$ in all calculations using various Skyrme interactions. The total angular momentum $J=60 \hbar$ consists of aligned 12 nucleons with the orbital angular momenta $\Lambda=$ $+4,+5$, and +6 for spin up-down neutrons and protons. The obtained results strongly suggest that a macroscopic amount of circulating current breaking the time-reversal symmetry emerges in the high-spin excited state of ${ }^{40} \mathrm{Ca}$.

PACS numbers: 21.60.Jz, 21.60.Ev, 27.40.+z
\end{abstract}

The investigations of nuclei rotating extremely fast about the symmetry axis provide a good opportunity to severely test the fundamental theory of a quantum mechanics. In a classical picture for such rotation, the nuclear density at the equatorial plane increases due to the strong centrifugal force, and the oblate deformations develop with increasing rotational frequencies [1]. However, such a collective rotation about the symmetry axis is quantum-mechanically forbidden. Instead, the spins and the orbital angular momenta of single particles are aligned with the symmetry axis building extremely high angular momenta [2, 4]. In this case, the total angular momentum of the nucleus is just the sum of the symmetry-axis components of those of the single particles. Bohr and Mottelson pointed out that in such a nucleus, a "macroscopic" amount of circulating current breaking the time-reversal symmetries emerges, which is a fascinating new form of the nuclear matter [3].

A typical example of such phenomena is the high-K oblate isomer states in ${ }^{152}$ Dy [5]. In the high angularmomentum states above $I=14 \hbar$, the observed excited states are irregularly distributed around the average yrast line, which is calculated by assuming a macroscopic nuclear shape with the strongly oblate deformations $(\beta \sim$ $-0.3)$. The emergence of these high-spin isomers around the yrast line can be naturally explained by the singleparticle alignments [3], because the E2 transitions, which are a characteristic quantity related to the rotational collectivity, are strongly forbidden for those nuclei. In addition, the $\gamma$ transitions are strongly suppressed due to the large difference of the internal structure between the initial and final states. Those high-spin isomers around the yrast line are thus called "yrast traps". Many experiments have been attempted to produce yrast traps with extremely high spins [6]. The observed highest angular momentum in this category is $I=49 \hbar$ in ${ }^{158} \mathrm{Er}$ [7].

In this Letter, we investigate the possibility of the existence of the torus configuration as an extreme limit of the high-spin oblate isomers. In such a limit, more nucleons with higher orbital angular momentum are aligned and its density is much denser at the equator part. We here consider a special case for the existence of the density only around its equator part. We below show that such exotic state can indeed exist at an extremely high angular momentum in ${ }^{40} \mathrm{Ca}$.

Many theoretical calculations have been performed to search for the high spin states with strongly oblate deformations in a wide range of nuclei. Those studies have been mainly focused on the nuclei with the oblate deformation at around $\beta \sim-0.3$ heavier than ${ }^{40} \mathrm{Ca}$ [4]. In contrast, Wong discussed the stability of the torus configuration using the macroscopic-microscopic model in heavymass systems [8-10]. The calculations using the constraint Hartree-Fock (-Bogoliubov) models have been also performed for heavy-mass systems [11, 12]. The calculations using $\alpha$ cluster model have been attempted to obtain the ring configurations consisting of alpha particles and extra neutrons [13]. However, as shown in the present study, an essential mechanism for the stability of such configuration is the extremely high angular momentum directed to the symmetry axis, resulting in the breaking of the timereversal symmetry in the intrinsic state.

To investigate the possibility of the existence of the torus configuration in ${ }^{40} \mathrm{Ca}$ with high angular momentum, we use the three-dimensional Skyrme Hartree-Fock (HF) method with a Lagrange multiplier, which is introduced to obtain the single-particle states aligned with the symmetry axis. To this end, we minimize the HF Hamiltonian, $\hat{H}$, with the Lagrange multiplier, $\omega$. In the present study, we take the 


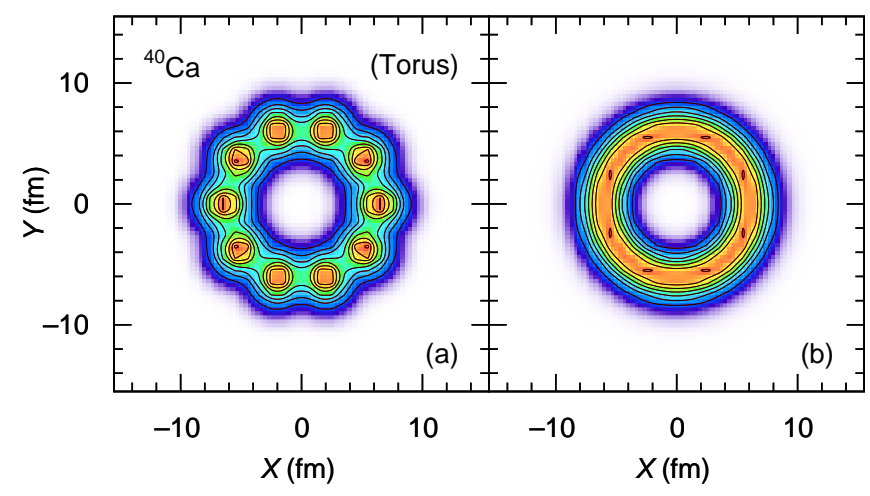

FIG. 1. (color online) Total density for (a) the initial condition of the HF iterations and (b) the calculated result with $\hbar \omega=1.5$ $\mathrm{MeV}$ at the $15000 \mathrm{HF}$ iterations. The density is integrated in the $z$ direction. The contours correspond to multiple steps of 0.05 $\mathrm{fm}^{-2}$. The color is normalized by the largest density in each plot.

$z$ axis as the symmetry axis. We first define the effective Hamiltonian, $\hat{H}^{\prime}$, given by $\hat{H}^{\prime}=\hat{H}-\omega \hat{J}_{z}$, where $\hat{J}_{z}$ denotes the operator for the sum of the $z$ components of the total angular-momentum for each single particle, $\hat{j}_{z}$, given by $\hat{J}_{z}=\sum_{i} \hat{j}_{z}^{(i)}$. In the HF approximation, $\hat{H}^{\prime}$ is rewritten as $\hat{H}^{\prime}=\sum_{i}\left\{\hat{h}_{i}-\omega j_{z}^{(i)}\right\}$, where $\hat{h}_{i}$ denotes the Hamiltonian for each single particle. The eigenvalue of $H^{\prime}$ is given by $\left\langle\hat{H}^{\prime}\right\rangle=\sum_{i}\left\{\left(e_{i}-\lambda\right)-\hbar \omega \Omega_{i}\right\}$, where $\lambda$ denotes the Fermi energy at $\omega=0$ and $e_{i}$ and $\Omega_{i}$ denote the energy and the $z$ component of the total angular momentum in the unit of $\hbar$ for each single particle, respectively. In the present study, we search for the stable state using the equivalent cranked Skyrme HF equation, $\delta\left\langle\hat{H}-\omega \hat{J}_{z}\right\rangle=0$ [14, 15], by scanning a large range of $\omega$.

Before the HF calculations, we here discuss the shell structure of the torus configuration using the radial displaced harmonic oscillator (RDHO) model [9]. For the torus configuration, not only $\Omega$ but also the $z$ component of the orbital angular momentum, $\Lambda$, are good quantum numbers $(\Omega=\Lambda+\Sigma$, where $\Sigma$ denotes the $z$ component of the spin values, $\pm 1 / 2$ ). Two nucleons in each $\Lambda$ energetically degenerate with the different spin values. At $\hbar \omega=0$, the lowest configuration for ${ }^{40} \mathrm{Ca}$ is $\Lambda=0, \pm 1, \pm 2, \pm 3$, and \pm 4 and the residual two nucleons can occupy any two states with $\Lambda= \pm 5$. At $\hbar \omega \neq 0$, the possible spin aligned configurations are (i) $\Lambda=0, \pm 1, \pm 2, \pm 3, \pm 4$ and +5 for the total angular momentum $J=20 \hbar[=5 \hbar \times 2$ (spin degeneracy) $\times 2$ (isospin degeneracy)], (ii) $\Lambda=0, \pm 1, \pm 2, \pm 3,+4,+5$ and +6 for $J=60 \hbar[=15 \hbar \times 2 \times 2]$, and (iii) $\Lambda=0, \pm 1$, $\pm 2,+3,+4,+5,+6$ and +7 for $J=100 \hbar[=25 \hbar \times 2 \times 2]$.

In the self-consistent calculations, the single-particle wave functions are described on a Cartesian grid with a grid spacing of $1.0 \mathrm{fm}$. We take $32 \times 32 \times 24$ grid points for the $x, y$, and $z$ directions, respectively. This was sufficiently accurate to provide converged configurations. The dampedgradient iteration method [16] is used, and all derivatives

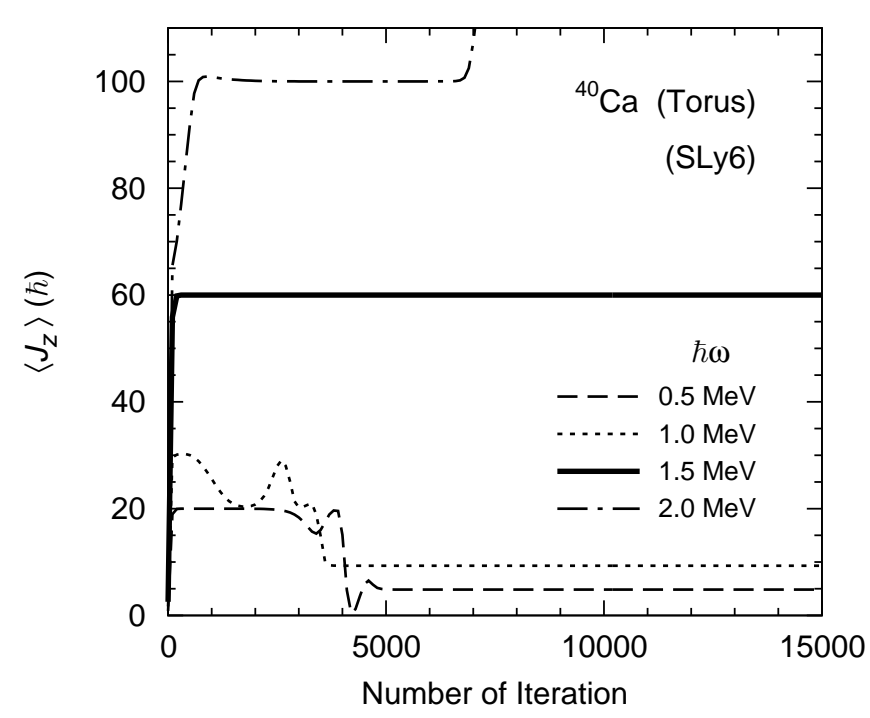

FIG. 2. Convergence behavior of the expectation value of $J_{z}$ in the HF calculations versus the number of iterations. The dashed, dash-dotted, solid, and dotted lines denote the calculated results with $\hbar \omega=0.5,1.0,1.5$, and $2.0 \mathrm{MeV}$, respectively.

are calculated using the Fourier transform method. We take three different Skyrme forces which all perform well concerning nuclear bulk properties but differ in details: SLy6 as a recent fit which includes information on isotopic trends and neutron matter [17], and SkI3 and SkI4 as recent fits especially for the relativistic isovector structure of the spinorbit force [18]. However, except for the effective mass, the bulk parameters (equilibrium energy and density, incompressibility, and symmetry energy) are comparable in the all interactions.

For the initial wave functions, we chose the ring configuration with $10 \alpha$ particles placed on the $x-y$ plane, as shown in Fig. 1(a). Each $\alpha$ particle is described by the Gaussian function with its center placed on $z=0$. Using this initial condition, we perform the HF iterations with 15000 times and investigate the convergence of the calculated results. Figure 2 shows the convergence behaviors of $\left\langle\hat{J}_{z}\right\rangle$ versus the number of the HF iterations with various $\omega$ 's using the SLy6 interaction. We can see that the result calculated with $\hbar \omega=1.5 \mathrm{MeV}$ converges rapidly to $J_{z}=60 \hbar$. Figure 1(b) shows the density obtained with $\hbar \omega=1.5 \mathrm{MeV}$ at the 15000th iteration step. The calculated result is indeed the torus configuration. The obtained density distribution, $\rho(r, z)$, can be well fitted by $\rho(r, z)=\rho_{0} e^{-\left\{\left(r-r_{0}\right)^{2}+z^{2}\right\} / \sigma^{2}}$, where $\rho_{0}=0.13 \mathrm{fm}^{-3}, r_{0}=6.07 \mathrm{fm}$, and $\sigma=1.61 \mathrm{fm}$ for the SLy6 interaction.

On the other hand, the calculated results with $\hbar \omega=0.5$ and $1.0 \mathrm{MeV}$ lead to unstable states. That for $\hbar \omega=2.0$ $\mathrm{MeV}$ leads to the fission. Although it seems that $\left\langle\hat{J}_{z}\right\rangle$ 's with $\hbar \omega=0.5$ and $1.0 \mathrm{MeV}$ converge at the 15000th step, those are in fact unstable. In Fig. 2, we can see that these states first converge to a quasi-stable state. After that, the 


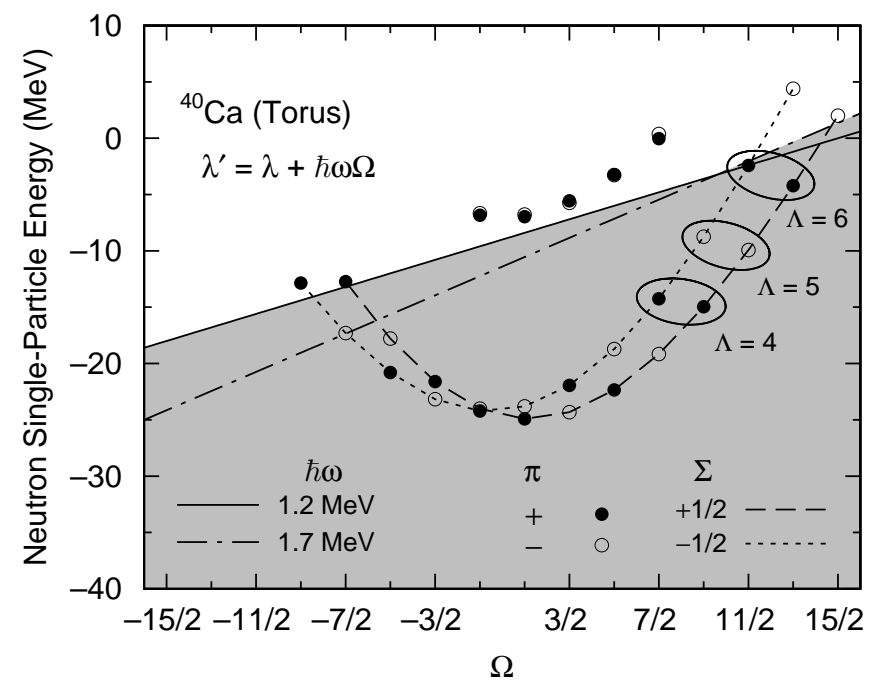

FIG. 3. Single-particle energies versus the $z$ component of the total angular momentum $\Omega$. The solid and open circle denote the single-particle states with the positive and the negative parties, respectively. The single-particle states with the $z$ component of the spin $\Sigma=+1 / 2$ and $-1 / 2$ are connected by the dashed and dotted lines, respectively. The solid and the dash-dotted lines denote the sloping Fermi energies with $\hbar \omega=1.2$ and $1.7 \mathrm{MeV}$, respectively. The gray area denotes the states below the Fermi energy.

instability of those states increases. In those quasi-stable states, $J_{z}$ is $20 \hbar$ for $\hbar \omega=0.5$, and $1.0 \mathrm{MeV}$ and is $100 \hbar$ for $\hbar \omega=2.0 \mathrm{MeV}$. Later, we will discuss those quasi-stable states with $J_{z}=20$ and $100 \hbar$.

We next investigate the region of the Lagrange multipliers where the torus configurations stabilize, using various Skyrme interactions. We find that such region of $\hbar \omega$ for the SLy6 and SkI3 interactions extends from 1.2 to $1.7 \mathrm{MeV}$. That for the SkI4 interaction is from 1.2 to $1.8 \mathrm{MeV}$. In such regions, we obtain the only one stable torus configuration at $J_{z}=60 \hbar$ in all calculations. The obtained excitation energies are $170.45,174.22$, and $172.53 \mathrm{MeV}$ for the SLy6, SkI3, and SkI4 interactions, respectively. The fitted radius of the torus configuration is almost the same for all calculations. The dependence of the calculated result on the choice of the interaction is weak. This is mainly because that the spin-orbit forces in the radial directions of the inner and outer surface of the torus configuration almost cancel out each other (see Eq. (4.14) in Ref. [9]). Thus, the RDHO mode is a good approximation for the present calculations.

Figure 3 shows the single-particle spectrum versus $\Omega$ calculated using the SLy6 interaction. The solid and open circles denote the single-particle states with the positive and the negative parities, respectively. The single-particle states with $\Sigma=+1 / 2$ and $-1 / 2$ are connected by the dashed and the dotted lines, respectively. These energies connected by each line are well proportional to $\Lambda^{2}$, which is consistent with that of the RDHO model [see Eq. (4.26) in Ref. [9]]. We here define the "sloping" Fermi energy, $\lambda^{\prime}$,

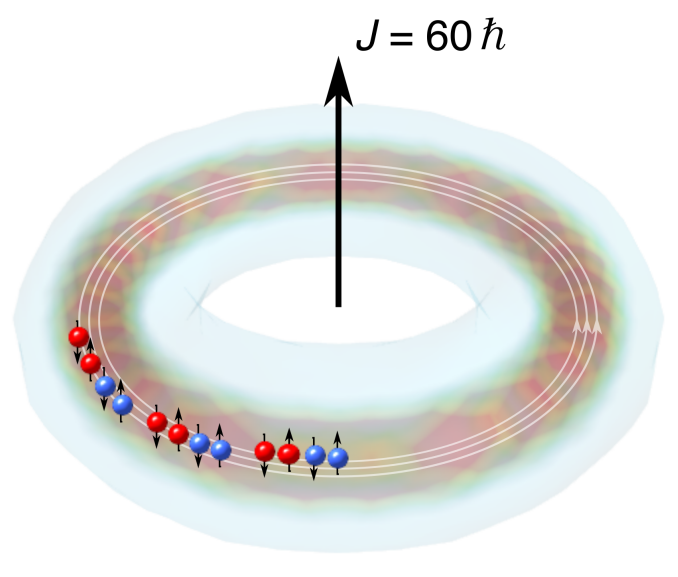

FIG. 4. (color online) Circulating currents of 12 nucleons. The outer layer of the torus surface denotes the half of the total density. The density plot in the inside of the torus surface denotes the calculated density of the aligned 12 single particles.

given by $\lambda^{\prime}=\lambda+\hbar \omega \Omega$ [4]. Then, the occupied states are obtained by $\left\langle\hat{H}^{\prime}\right\rangle=\sum_{i}\left(e_{i}-\lambda^{\prime}\right)$ with $e_{i}<\lambda^{\prime}$. The solid and the dash-dotted lines denote the sloping Fermi energies with $\hbar \omega=1.7$ and $1.2 \mathrm{MeV}$, respectively. The occupied states are denoted by the gray area. In Fig. 3, we can clearly see that the torus configuration can exist at $J_{z}=60$ $\hbar$ in the region from $\hbar \omega=1.2$ to $1.7 \mathrm{MeV}$. The total angular momentum of $J_{z}=60 \hbar$ consists of the spin-up-down pairs of the aligned single particles with the same $\Lambda,\left(\Omega_{+}\right.$, $\left.\Omega_{-}\right)$, of $\left(+9 / 2^{+},+7 / 2^{+}\right)$with $\Lambda=4,\left(+11 / 2^{-},+9 / 2^{-}\right)$with $\Lambda=5$, and $\left(+13 / 2^{+},+11 / 2^{+}\right)$with $\Lambda=6$ (see the solid ellipses in Fig. 3).

As discussed in the shell structure of the torus configuration, the states with $J=20,60$, and $100 \hbar$ are the possible combinations of the spin alignment for the RDHO model. For the quasi-stable state with $J=20 \hbar$, the centrifugal force is insufficient to stabilize the torus configuration against the strong nuclear attractive force. On the other hand, to build the state with $J=100 \hbar$, the unbound states ( $e>0$ ) with $\Omega=+15 / 2$ and $+11 / 2$ are occupied, resulting in the instability for the torus configuration. Only for $J=60 \hbar$, the torus configuration can be stabilized. Thus, the stability of the state with $J=60 \hbar$ is rather robust. Although we have performed the similar calculations for ${ }^{24} \mathrm{Mg}$ and ${ }^{32} \mathrm{~S}$, we could not find the stable torus states for those nuclei.

As shown in the present study, if the state with the torus configuration is formed at $J=60 \hbar$, the macroscopic circulating current of 12 nucleons with $\Lambda=+4,+5$, and +6 strongly violates the time-reversal symmetry in the intrinsic state (see Fig. 4). It is interesting to investigate how this fascinating new state can be observed in experiments. The state with the torus configuration at $J=60 \hbar$ would have an extremely large magnetic moment $\left(\mu=30 \mu_{N}\right)$. This 
would lead to a procession motion under an external magnetic field.

A question then arises how such a "femto-scale magnet" rotates spontaneously. When the spherical symmetry is broken, the collective rotation emerges spontaneously, in principle, to restore the broken symmetry. However, it is unclear whether such a state built with significant amount of circulating current can rotate about the perpendicular direction to the symmetry axis or not. If not, such a state would be an anomalous one, which has not yet been recognized in the experiments. Even if the state can rotate, the rotational band built on such a state would show interesting behaviors for their M1/E2 transition strengths and moment of inertia, $\mathscr{T}_{\perp}$. It is intriguing to investigate the extent of the difference between the experimental and the classical rigid-body moments of inertia $\left(\mathscr{T}_{\perp}=20.97 \hbar^{2} \cdot \mathrm{MeV}^{-1}\right.$ in the case of the SLy6 interaction).

To identify the torus configuration in the experiment, it is also important to discuss the competition to the fission decay channel. The calculation using the macroscopic model with the FRLDM2002 parameter set [19] shows that the fission barrier for the spheroidal deformations vanishes at $J=32 \hbar$ and an excitation energy of $49.27 \mathrm{MeV}$ [20]. Our calculated results for the torus configuration are considerably higher than those values. However, it would be possible that the state with the torus configuration can survive against the fission. One important example for such possibility is the long-lived $K^{\pi}=16^{+}$isomer in ${ }^{178} \mathrm{Hf}$ with a half-lives of about 31 y [21], which is extremely long compared to other isomers (4 $\mathrm{s}$ for the $K^{\pi}=8^{-}$isomer). In this connection, it is unclear how the collective path from a topologically different torus configuration is connected to the region of the spheroidal deformations leading to the fission [10, 12]. Wong indeed showed that the torus configurations are stable from $J \sim 57$ to $74 \hbar$ for mass number $A \sim 50$ [10].

In summary, we have suggested the existence of the torus configuration in the extremely high-spin excited states of ${ }^{40} \mathrm{Ca}$ using the three-dimensional cranked Skyrme HF method. We found only one stable state with the torus configuration at $J_{z}=60 \hbar$ in all the calculations with any Skyrme interactions. The calculated excitation energies of this state are $170.45,174.22$, and $172.53 \mathrm{MeV}$ for the SLy6, SkI3, and SkI4 interactions, respectively. The obtained results are insensitive to the choice of the Skyrme interactions, because the contribution of the spin-orbit force is small in the torus configuration. To build the torus state with $J=60 \hbar$, the 6 nucleons for $\Omega=+9 / 2^{+}$and $+7 / 2^{+}$ with $\Lambda=4, \Omega=+11 / 2^{-}$and $+9 / 2^{-}$with $\Lambda=5$, and $\Omega=+13 / 2^{+}$and $+11 / 2^{+}$with $\Lambda=6$ are aligned with the symmetry axis for both protons and neutrons. We have shown that such configuration is built on the major shell structure estimated by the RDHO model for the rotating torus shape, indicating that the torus state is robustly stable and the macroscopic amount of the time-reversal symmetry breaking occurs. Although the observation of the torus state would be difficult, exploration for such an exotic state would provide us valuable information on the new frontier of the nuclear matter, which is a big challenge both theoretically and experimentally.

A part of this research has been funded by MEXT HPCI STRATEGIC PROGRAM. This work was undertaken as part by the Yukawa International Project for Quark-Hadron Sciences (YIPQS), and was partly supported by the GCOE program "The Next Generation of Physics, Spun from Universality and Emergence" from MEXT of Japan. J.A. M. and P.-G. R. were supported by BMBF under contract numbers 06ER9063 und 06FY9086, respectively. One of the authors (JAM) would like to thank the Japan Society for the Promotion of Science (JSPS) for an invitation fellowship for research in Japan.

[1] S. Cohen, F. Plasil and W.J. Świa̧tecki, Ann, Phys. 82, 557 (1974).

[2] A. Bohr, Proc. Int. Physics 'Enrico Fermi', Cours LXIX, ed. A. Bohr and R. A. Broglia (Amsterdam: North-Holland, 1977), p3.

[3] A. Bohr and B.R. Mottelson, Nucl. Phys. A354, 303c (1981).

[4] A.V. Afanasjev, D.B. Fossan, G.J. Lane, and I. Ragnarsson, Phys. Rep. 322, 1 (1999).

[5] T.L. Khoo et al., Phys. Rev. Lett. 41, 1027 (1978).

[6] E.S. Paul et al., Phys. Rev. Lett. 98, 012501 (2007).

[7] J. Simpson et al., Phys. Lett. B327, 187 (1994).

[8] C.Y. Wong, Phys. Lett. 41B, 446 (1972).

[9] C.Y. Wong, Ann. Phys. 77, 279 (1973).

[10] C.Y. Wong, Phys. Rev. C 17, 331 (1978).

[11] M. Warda, Int. J. Mod. Phys. E16, 452 (2007)

[12] A. Staszczak and C.Y. Wong, Act. Phys. Pol. B 40, 753 (2009)

[13] D.H. Wilkinson, Nucl. Phys. A 452, 296 ( 1986).

[14] J.A. Maruhn et al., Phys. Rev. C 74, 044311 (2006).

[15] T. Ichikawa et al., Phys. Rev. Lett. 107, 112501 (2011).

[16] P.-G. Reinhard, and R. Y. Cusson, Nucl. Phys. A378, 418 (1982).

[17] E. Chabanat, P. Bonche, P. Haensel, J. Meyer, and R. Schaeffer, Nucl. Phys. A627, 710 (1997).

[18] P.-G. Reinhard and H. Flocard, Nucl. Phys. A584, 467 (1995).

[19] P. Möller, A. J. Sierk, and A. Iwamoto, Phys. Rev. Lett. 92 (2004) 072501.

[20] A.J. Sierk, Phys. Rev. C 33, 2039 (1986); private communications.

[21] T.L. Khoo, F.M. Bernthal, R.G.H. Robertson, and R.A. Warner, Phys. Rev. Lett. 37, 823 (1976). 